\title{
Interaction of Different Water Amounts and Phosphorus and Magnesium Doses and Their Effects on Water-Yield Relations of Soybean (Glycine max L.) in Arid Conditions
}

\author{
Aise DELIBORAN ${ }^{1}$, Erdal SAKIN², Hasan ASLAN ${ }^{1}$, Ahmet Ruhi MERMUT² \\ ${ }^{1}$ GAP Soil-Water Resources and Agricultural Researches Institute, Boulevard Ucaksavar, 63040 Sanliurfa, Turkey; aisedeliboran@gmail.com \\ ${ }^{2}$ Harran University, Agriculture of Faculty, Department of Soil Science and Plant Nutrition, Campus of Osmanbey, 63030 Sanliurfa, Turkey
}

\begin{abstract}
This field experiment examined the effects of magnesium $(\mathrm{Mg})$ and phosphorus $(\mathrm{P})$ fertilizers, which were given in different doses to soybean grown in different water levels, on plant water consumption, water-yield relations and grain yield factors. The experiment used a random blocks designed with three consecutive and was conducted over two years 2006 and 2007 . Four different $P$ doses $(0-4-8-12 \mathrm{~kg}$ $\left.\mathrm{P} \mathrm{da}^{-1}\right)$ and three different $\mathrm{Mg}$ doses $\left(0-4-8 \mathrm{~kg} \mathrm{Mg} \mathrm{da}^{-1}\right)$ were applied with three different water levels $\left(\mathrm{I}_{1}, \mathrm{I}_{2}, \mathrm{I}_{3}\right)$. Cumulative pan values obtained from Class A Pan evaporation pool were applied as irrigation (I) $33 \%$ for $\mathrm{I} 1,67 \%$ for $\mathrm{I}_{2}, 100 \%$ for $\mathrm{I}_{3}$. At the end of experiment were determined plant water consumption, water-yield relations and grain yield. At the start of irrigation, $548.00 \mathrm{~mm}, 786.00 \mathrm{~mm}$ and 1 $017.00 \mathrm{~mm}$ of water were given to $I_{1}, I_{2}$ and $I_{3}$ plots, respectively in the first year, and $457.98 \mathrm{~mm}, 698.02 \mathrm{~mm}$ and $931.00 \mathrm{~mm}$ irrigation water was given in the second year. Plant water consumption values $\left(\mathrm{ET}_{\mathrm{z}}\right)$ were determined as $648.00 \mathrm{~mm}, 903.00 \mathrm{~mm}$ and $1164.00 \mathrm{~mm}$ for $\mathrm{I}_{1}, \mathrm{I}_{2}$ and $\mathrm{I}_{3}$ parts in the first year, and $567.00 \mathrm{~mm}, 825.00 \mathrm{~mm}$ and $1070.00 \mathrm{~mm}$ in the second year. Moreover, according to experiment plots, irrigation water treatment efficiency (IWTE) varied between 0.14 and $0.53 \mathrm{~kg} \mathrm{~m}^{-3}$ in the first year and between 0.16 and $0.59 \mathrm{~kg}$ $\mathrm{m}^{-3}$ in the second year.
\end{abstract}

Keywords: magnesium, phosphorus, plant water consumption, soybean, water limitation, water-yield relation

Abbreviation: da-decare; ha-hectare; GAP-Southeast Anatolia Project; IWTE-Irrigation Water Treatment Efficiency; KHGM-Koy Hizmetleri Genel Mudurlugu; L-Litter; Mg-Magnesium; N-Nitrogen; P-Phosphorus; SAR-Southeast Anatolia Region; t-Tonne; TSMSTurkish State Meteorological Service; WCE-Water Consumption Efficiency; WUE-Water Usage Efficiency

\section{Introduction}

In addition to plant nutrition elements, plant type and species, another important factor for yield and quality of plant yield is water. Moreover, one of the biggest problems of almost all countries the sustainable use of soil and water sources. Because water sources on earth are finite. As the Southeast Anatolia Region (SAR) is in an arid and semiarid climate region and the water needs of plants are not met by natural precipitation, the importance of irrigation is increasing. On the SAR Plains, despite appropriate soil resources and the other climatic factors, inadequate precipitation in spring and summer months is the primary factor affecting agricultural development within the region. To prevent water inadequacy, which is the primary factor limiting plant types grown on the SAR Plains, the yield obtained from each area and modern agriculture methods applications is an accelerative, encouraging and fascinating element which will increase agriculture yield, employment facilities, other sectors and services (Tekinel, 1998). All studies conclude that products lost seen during the season are the consequence of water inadequacy. It is suggested that appropriate irrigation programs are developed, to minimize yield losses (Tulucu, 1985).

Shariatmadari and Mermut (1999), found that clay mineral with sepiolite silicate releases high levels of $\mathrm{Mg}$ to the environment and increases $\mathrm{P}$ resolution. Previous studies found that $P$ function can be increased by applying $\mathrm{Mg}$ to the soil, resulting in increased $\mathrm{P}$ desorption at the environment with $\mathrm{Mg}$. This finding has great importance for the uptake of P by plants on calcareous soils. With the main focus of the present study is the effect of $\mathrm{Mg}$, taking $P$ from soil at different irrigation levels, and so increasing yields. For this reason, different magnesium and phosphorus doses and water limitations were applied to soybean plants.

The study reviews the outcomes of different water limitations and $\mathrm{P}$ and $\mathrm{Mg}$ doses to determine the implications for irrigation water amounts, plant water consumption values, irrigation water treatment efficiency (IWTE), water consumption efficiency (WCE), rational water consumption $\left(\mathrm{ET}_{\mathrm{a}} / \mathrm{ET}_{\mathrm{m}}\right)$, rational plant water decrease $\left(1-\left(\mathrm{ET}_{\mathrm{a}} / \mathrm{ET}_{\mathrm{m}}\right)\right)$, rational yield $\left(\mathrm{Y}_{\mathrm{a}} / \mathrm{Y}_{\mathrm{m}}\right)$ and rational yield decrease $\left(1-\left(Y_{a} / Y_{m}\right)\right.$. 
184

\section{Materials and methods}

This study was conducted at Harran University Agriculture Faculty Campus during 2006 and 2007. The study investigated soybean (Glycine max L.) cultivar 'Nova'. The study site is located at $36^{\circ} 42^{\prime} \mathrm{N}$ latitude and $38^{\circ} 58^{\prime} \mathrm{E}$ longitudes and its altitude is $464 \mathrm{~m}$.

The research area is in the SAR climate region and is influenced by the Mediterranean climate. It is hot and drought-prone in summer and warm in winter. In 2006, between June and October, which includes the soybean growth period, mean temperature ranged between 20.6$30.8^{\circ} \mathrm{C}$, mean minimum temperature ranged between $12.8-22.8^{\circ} \mathrm{C}$, mean precipitation was $0-0.3 \mathrm{~mm}$, mean relative humidity was between $40.8-61.5 \%$, and soil temperature was $23.9-36.6^{\circ} \mathrm{C}$. In 2007 , mean temperature ranged between $21.6-30.4^{\circ} \mathrm{C}$, mean minimum temperature was 16.5-23. $0^{\circ} \mathrm{C}$, mean precipitation was $0.8-25.9 \mathrm{~mm}$, mean relative humidity ranged between $36.9-47.7 \%$, and soil temperature was between $23.3-35.0^{\circ} \mathrm{C}$ (Tab. 1).

The research was used a complete randomized block design (CRBD) methodology with three replications. Irrigation treatments were the main plot and $\mathrm{P}$ and $\mathrm{Mg}$ subjects were sub-plot. Four different $\mathrm{P}$ doses (0-4-8-12 kg P $\left.\mathrm{da}^{-1}\right)$ and three different $\mathrm{Mg}$ doses $\left(0-4-8 \mathrm{~kg} \mathrm{Mg} \mathrm{da}^{-1}\right)$ were applied with three different water levels $\left(\mathrm{I}_{1}, \mathrm{I}_{2}, \mathrm{I}_{3}\right)$. Cumulative pan values obtained from Class A Pan evaporation pool were applied as $33 \%$ for $I_{1}, 67 \%$ for $I_{2}, 100 \%$ for $I_{3}$. Phosphorus treatments were determined as $\mathrm{P}_{0}(0 \mathrm{~kg} \mathrm{P}$ da $\left.{ }^{1}\right), \mathrm{P}_{1}\left(4 \mathrm{~kg} \mathrm{P} \mathrm{da}^{-1}\right), \mathrm{P}_{2}\left(8 \mathrm{~kg} \mathrm{P} \mathrm{da}^{-1}\right), \mathrm{P}_{3}\left(12 \mathrm{~kg} \mathrm{P} \mathrm{da}^{-1}\right)$; and $\mathrm{Mg}$ treatments were determined as $\mathrm{Mg}_{0}\left(0 \mathrm{~kg} \mathrm{Mg} \mathrm{da}^{-1}\right)$, $\mathrm{Mg}_{1}\left(4 \mathrm{~kg} \mathrm{Mg} \mathrm{da}^{-1}\right)$ and $\mathrm{Mg}_{2}\left(8 \mathrm{~kg} \mathrm{Mg} \mathrm{da}^{-1}\right)$.

In the SAR, high soil temperature is an important factor in soybean production as a second yield, as it restricts bacteria responsible for Nitrogen-fixation. Therefore, bacteria inoculation was not performed during the research. Nitrogen fertilizer was given to plants as ammonium sulfate $\left(\left(\mathrm{NH}_{4}\right)_{2} \mathrm{SO}_{4}\right)$ at a dosage of $10 \mathrm{~kg} \mathrm{~N} \mathrm{da}^{-1}$. $\mathrm{N}$ fertilizer was applied in two stages, with the first part given during cultivation and remainder given equally to all parcels before flowering. Phosphorus and Mg treatments were applied to the experiment plot in the same way as to each irrigation part, in the combinations are below:

$\begin{array}{llll}\mathrm{P}_{0} \mathrm{Mg}_{0} & \mathrm{P}_{1} \mathrm{Mg}_{0} & \mathrm{P}_{2} \mathrm{Mg}_{0} & \mathrm{P}_{3} \mathrm{Mg}_{0} \\ \mathrm{P}_{0} \mathrm{Mg}_{1} & \mathrm{P}_{1} \mathrm{Mg}_{1} & \mathrm{P}_{2} \mathrm{Mg}_{1} & \mathrm{P}_{3} \mathrm{Mg}_{1} \\ \mathrm{P}_{0} \mathrm{Mg}_{2} & \mathrm{P}_{1} \mathrm{Mg}_{2} & \mathrm{P}_{2} \mathrm{Mg}_{2} & \mathrm{P}_{3} \mathrm{Mg}_{2}\end{array}$

In the study was used triple super phosphate as the $\mathrm{P}$ source and magnesium sulfate $\left(\mathrm{MgSO}_{4} .7 \mathrm{H}_{2} \mathrm{O}\right)$ as the $\mathrm{Mg}$ source; these were applied to plot during cultivation like being the equivalent to plant order and not being two fertilizer consecutively. Irrigation system was set up and the first water was given to the plants. Partial irrigation started, after exiting finished and proper moisture decreased to $40 \%$ levels on soil.

A drip irrigation system was set up and the first water was applied after sowing and fertilizer treatments. Ir- rigation started on 14.07.2006 in the first year and on 07.07.2007 in the second year, after exiting finished and proper moisture decreased to $40 \%$ levels on soil. Four days were chosen as the irrigation sequence. Moreover, the drip irrigation system was designed so that it wet all of the experimental area. To apply the system, water was taken from a source with a pump, after passed from a control unit; the water was applied at a pressure of $1.5 \mathrm{~atm}$. with $4 \mathrm{~L} \mathrm{~h}^{-1}$ flow on the main pipe, manifold and laterals. Emitters with a $50 \mathrm{~cm}$ sequence of drippers were preferred on positioned on laterals.

Evaporation values were measured on a Class A Pan evaporation pot. In both years of the experiment to determine the moisture content of the soil, soil samples were taken from $0-30 \mathrm{~cm}, 30-60 \mathrm{~cm}$ and $60-90 \mathrm{~cm}$ before and after irrigation started. Their wet weight was calculated and then they were placed in a drying closet at $105^{\circ} \mathrm{C}$ until the weight stabilized; the dry weight of the soils samples was then calculated. Plants benefit from water in different ways, according to the amount of water available amounts in their roots, and yields are shaped according to the moisture content of soils (Yaron, 1971). Water-yield function is based on this theory and this relationship occurs as a polynomial on cultivars (Kadayifci, 1996). During the growth season, under limited water conditions, the Stewart model in Eq. 1 was used to calculate rational yield decrease and rational water consumption decline (Doorenbos and Kassam, 1988).

$$
1-\frac{Y_{a}}{Y_{m}}=k y\left(1-\frac{E T_{a}}{E T_{m}}\right)
$$

$$
\begin{aligned}
& \text { Where: } Y_{a}: \text { true yield }\left(\mathrm{kg} \mathrm{da}^{-1}\right), \\
& Y_{m}: \text { maximum yield }\left(\mathrm{kg} \mathrm{da}^{-1}\right), \\
& k_{d}: \text { yield reaction factor, } \\
& E T_{a}: \text { true water consumption }(\mathrm{mm}) \text { and } \\
& E T_{m}: \text { maximum plant water consumption }(\mathrm{mm}) \\
& \frac{Y_{a}}{Y_{m}}: \text { is rational yield decrease } \\
& \frac{E T_{a}}{E T_{m}}: \text { is rational water consumption } \\
& 1-\frac{Y_{a}}{Y_{m}}: \text { denotes rational water decrease } \\
& 1-\frac{E T_{a}}{E T_{m}}: \text { shows rational water consumption decline }
\end{aligned}
$$

Water amounts in irrigation programs $(\mathrm{mm})$, yield values, irrigation water treatment efficiency (IWTE) and water usage efficiency (WUE) were calculated using the method of Howell et al. (1990), and rational water consumption $\left(\mathrm{ET}_{\mathrm{a}} / \mathrm{ET}_{\mathrm{m}}\right)$, rational plant water decline (1$\left.\left(\mathrm{ET}_{\mathrm{a}} / \mathrm{ET}_{\mathrm{m}}\right)\right)$, rational yield $\left(\mathrm{Y}_{\mathrm{a}} / \mathrm{Y}_{\mathrm{m}}\right)$ and rational yield decrease $\left(1-\left(Y_{\mathrm{a}} / \mathrm{Y}_{\mathrm{m}}\right)\right.$ results were calculated according to the methods of Doorenbos and Kassam (1988). 
Tab.1. Climate parameters of Sanliurfa in 2006 and 2007 (TSMS, 2006; 2007)

\begin{tabular}{|c|c|c|c|c|c|c|c|c|}
\hline Years & Months & $\begin{array}{l}\text { Mean } \max \\
\text { temp. }\left({ }^{\circ} \mathrm{C}\right)\end{array}$ & $\begin{array}{l}\text { Mean min } \\
\text { temp. }\left({ }^{\circ} \mathrm{C}\right)\end{array}$ & $\begin{array}{c}\text { Mean temp. } \\
\left({ }^{\circ} \mathrm{C}\right)\end{array}$ & $\begin{array}{c}\text { Mean } \\
\text { humidity }(\%) \\
\end{array}$ & $\begin{array}{c}\text { Mean } \\
\text { precipitation }(\mathrm{mm})\end{array}$ & $\begin{array}{l}\text { Sunbeam } \\
\left.\mathrm{cal} / \mathrm{cm}^{2}\right)\end{array}$ & $\begin{array}{l}5 \mathrm{~cm} \text { soil } \\
\text { Temp. }\left({ }^{\circ} \mathrm{C}\right)\end{array}$ \\
\hline \multirow{5}{*}{2006} & June & 38.0 & 22.8 & 30.8 & 40.8 & 0.3 & 605.1 & 36.6 \\
\hline & July & 38.5 & 24.9 & 32.2 & 45.5 & 0.3 & 560.5 & 38.8 \\
\hline & August & 40.4 & 26.0 & 33.4 & 44.6 & - & 462.2 & 39.0 \\
\hline & September & 32.3 & 22.4 & 27.2 & 42.3 & - & 455.4 & 32.6 \\
\hline & October & 25.9 & 12.8 & 20.6 & 61.5 & 42.5 & 291.7 & 23.9 \\
\hline \multirow{5}{*}{2007} & June & 37.2 & 23.0 & 30.4 & 36.9 & 0.8 & 614.2 & 35.0 \\
\hline & July & 40.8 & 27.0 & 34.0 & 31.3 & 8.0 & 553.2 & 38.5 \\
\hline & August & 39.3 & 25.4 & 32.2 & 41.9 & 3.2 & 521.4 & 37.4 \\
\hline & September & 36.0 & 22.0 & 28.4 & 36.4 & - & 427.3 & 32.6 \\
\hline & October & 28.4 & 16.5 & 21.6 & 47.7 & 25.9 & 320.6 & 23.3 \\
\hline \multirow{5}{*}{$\begin{array}{l}\text { Long-term } \\
\text { Mean } \\
(1929-2008)\end{array}$} & June & 44.0 & 8.3 & 27.9 & 32.4 & 3.0 & 583.1 & 32.6 \\
\hline & July & 46.8 & 15.0 & 33.1 & 29.6 & 0.6 & 561.9 & 37.2 \\
\hline & August & 46.2 & 15.5 & 31.2 & 32.3 & 0.9 & 513.0 & 36.0 \\
\hline & September & 42.0 & 0.0 & 26.7 & 35.1 & 1.1 & 436.0 & 30.6 \\
\hline & October & 37.8 & 1.9 & 20.1 & 44.8 & 23.8 & 315.1 & 21.9 \\
\hline
\end{tabular}

\section{Results and discussion}

Magnesium applications raise $\mathrm{P}$ resolutions in soil with the effect of water. As $\mathrm{Mg}$ resolves $\mathrm{P}$ and facilitates uptake by plants, the amounts of P-fertilizer can be reduced, thereby fertilizer costs are reduced. The findings show how $\mathrm{Mg}$ is effective in cases of reduced irrigation and fertilizing treatment and represent a new treatment in fertilizing. Moreover, limiting irrigation to some extent, with the aim of conserving water sources, applying water limitation programs, producing plant strains that are resistant to water stress, and determining the appropriate irrigation facilities for these kinds of plants are important for this region.

It is understood that with the start of irrigation on Harran Plain, soybean can be included in crop-rotations. The results show that high quality yield can be obtained with water and fertilizer savings; these results should be conveyed to producers and the findings may inform further studies of soybean in this region. The $90 \mathrm{~cm}$ effective root depth of soybean was taken into consideration for irrigation water levels and plant water consumption. After all irrigation parts were put on the field level, partial irrigation started on 14.07.2006 in the first year (Fig. 1) and on 07.07.2007 in the second year (Fig. 2).

From germination time to the beginning of irrigation, $317.00 \mathrm{~mm}$ irrigation water was applied to all parts in the first year and $225.00 \mathrm{~mm}$ in the second year. With the beginning of partial irrigation, $231.00 \mathrm{~mm}, 469.00 \mathrm{~mm}$ and $700.00 \mathrm{~mm}$ irrigation water was applied to $\mathrm{I}_{1}, \mathrm{I}_{2}$ and $\mathrm{I}_{3}$ parts, respectively, in the first year, and $232.98 \mathrm{~mm}, 473.02$ $\mathrm{mm}$ and $706.00 \mathrm{~mm}$ in the second year. For the same parts, plant water consumption values were calculated as 648.00 , 903.00 and $1164.00 \mathrm{~mm}$, respectively, in the first year and $567.00,825.00$ and $1070.00 \mathrm{~mm}$ in the second year. Statistically significant difference was not found in plant water consumption values between the two study years. It is thought that the observed difference was due to variations in climatic factors between years. The FAO (1979), stated that, for maximum yield, the seasonal water need of soybean is between $450-700 \mathrm{~mm}$ according to climate and length of growing season and that irrigation in critical growth periods greatly increases the yield. Gercek et al. (2003), reported that total amounts of water applied to soy by sprinkling and dripping irrigation methods were 1295 $\mathrm{mm}$ in the first year and $1369 \mathrm{~mm}$ in the second year, and measured plant water consumption values with dripping irrigation were $1378 \mathrm{~mm}$ in the first year and $1509 \mathrm{~mm}$ in the second year. Ozkara (1991), found that, during the irrigation season the maximum yield was obtained from the irrigation part on which $60 \%$ of moisture was consumed; this irrigation part required $334.2 \mathrm{~mm}$ irrigation water, its seasonal water consumption was $444.9 \mathrm{~mm}$ and its average seasonal water consumption quotient was (q) 0.54 .

IWTE and WCE created important difference in the relationship between applied water amounts and yield. Although the irrigation levels were the same, the highest yield was obtained from $\mathrm{I}_{1}$ part of $\mathrm{P}_{3} \mathrm{Mg}_{2}$ treatment. The same results were applicable for 2007, and were calculated as 0.68 and $0.66 \mathrm{~kg} \mathrm{~m}^{-3}$, respectively. Water consumption efficiency was like the irrigation water treatment efficiency in 2006 and 2007 (Tab. 2).

When irrigation water amount was applied two fold of $\mathrm{I}_{1}$ irrigation part amount on the $\mathrm{P}_{2} \mathrm{Mg}_{2}$ fertilizer combination in two years respectively 0.63 and $0.60 \mathrm{~kg} \mathrm{~m}^{-3}$ IWTE occurred. Moreover, the water consumption efficiency (WCE) amounts were calculated as 0.52 and 0.54 $\mathrm{kg} \mathrm{m}^{-3}$, respectively. Although the amount of water was the same $(836 \mathrm{~mm})$ in these two efficiency values, IWTE was 0.17 for the first year and $0.18 \mathrm{~m}^{-3}$ for the second year; and WCP in the two study years was 0.14 and $0.16 \mathrm{~kg} \mathrm{~m}^{-3}$, respectively (Tab. 2). 
Tab. 2. Irrigation water amounts, plant water consumption (ET), irrigation water treatment (IWTE) and water consumption efficiency (WCE) values (2006-2007)

\begin{tabular}{|c|c|c|c|c|c|c|c|c|c|c|}
\hline \multirow[t]{2}{*}{ Irrigation Unit } & \multicolumn{2}{|c|}{$\begin{array}{c}\text { Amounts of irrigation } \\
\text { water }(\mathrm{mm})\end{array}$} & \multicolumn{2}{|c|}{$\begin{array}{c}\text { Plant water } \\
\text { consumption } \mathrm{ET}_{2}(\mathrm{~mm})\end{array}$} & \multicolumn{2}{|c|}{$\begin{array}{c}\text { Yield } \\
\left(\mathrm{kg} \mathrm{da}^{-1}\right)\end{array}$} & \multicolumn{2}{|c|}{$\begin{array}{l}\text { IWTE } \\
\left(\mathrm{kg} \mathrm{m}^{-3}\right)\end{array}$} & \multicolumn{2}{|c|}{$\begin{array}{c}\text { WCE } \\
\left(\mathrm{kg} \mathrm{m}^{-3}\right)\end{array}$} \\
\hline & 2006 & 2007 & 2006 & 2007 & 2006 & 2007 & 2006 & 2007 & 2006 & 2007 \\
\hline $\mathrm{S}_{1}-\mathrm{P}_{0} \mathrm{Mg}_{0}$ & 598.00 & 507.98 & 648.00 & 567.00 & 111.07 & 107.40 & 0.19 & 0.21 & 0.17 & 0.19 \\
\hline $\mathrm{S}_{1}-\mathrm{P}_{0} \mathrm{Mg}_{1}$ & 598.00 & 507.98 & 648.00 & 567.00 & 126.83 & 125.50 & 0.25 & 0.25 & 0.20 & 0.22 \\
\hline $\mathrm{S}_{1}-\mathrm{P}_{0} \mathrm{Mg}_{2}$ & 598.00 & 507.98 & 648.00 & 567.00 & 132.10 & 131.93 & 0.26 & 0.26 & 0.20 & 0.23 \\
\hline $\mathrm{S}_{1}-\mathrm{P}_{1} \mathrm{Mg}_{0}$ & 598.00 & 507.98 & 648.00 & 567.00 & 134.73 & 121.30 & 0.27 & 0.24 & 0.21 & 0.21 \\
\hline $\mathrm{S}_{1}-\mathrm{P}_{1} \mathrm{Mg}_{1}$ & 598.00 & 507.98 & 648.00 & 567.00 & 140.77 & 140.17 & 0.28 & 0.28 & 0.22 & 0.25 \\
\hline $\mathrm{S}_{1}-\mathrm{P}_{1} \mathrm{Mg}_{2}$ & 598.00 & 507.98 & 648.00 & 567.00 & 162.00 & $156 ., 53$ & 0.32 & 0.31 & 0.25 & 0.28 \\
\hline $\mathrm{S}_{1}-\mathrm{P}_{2} \mathrm{Mg}_{0}$ & 598.00 & 507.98 & 648.00 & 567.00 & 207.80 & 219.83 & 0.41 & 0.43 & 0.32 & 0.39 \\
\hline $\mathrm{S}_{1}-\mathrm{P}_{2} \mathrm{Mg}_{1}$ & 598.00 & 507.98 & 648.00 & 567.00 & 217.40 & 239.57 & 0.43 & 0.47 & 0.34 & 0.42 \\
\hline $\mathrm{S}_{1}-\mathrm{P}_{2} \mathrm{Mg}_{2}$ & 598.00 & 507.98 & 648.00 & 567.00 & 235.07 & 250.17 & 0.46 & 0.49 & 0.36 & 0.44 \\
\hline $\mathrm{S}_{1}-\mathrm{P}_{3} \mathrm{Mg}_{0}$ & 598.00 & 507.98 & 648.00 & 567.00 & 188.00 & 219.87 & 0.37 & 0.43 & 0.29 & 0.39 \\
\hline $\mathrm{S}_{1}-\mathrm{P}_{3} \mathrm{Mg}_{1}$ & 598.00 & 507.98 & 648.00 & 567.00 & 243.50 & 243.40 & 0.48 & 0.48 & 0.38 & 0.43 \\
\hline $\mathrm{S}_{1}-\mathrm{P}_{3} \mathrm{Mg}_{2}$ & 598.00 & 507.98 & 648.00 & 567.00 & 345.17 & 334.17 & 0.68 & 0.66 & 0.53 & 0.59 \\
\hline $\mathrm{S}_{2}-\mathrm{P}_{0} \mathrm{Mg}_{0}$ & 836.00 & 748.02 & 903.00 & 825.00 & 128.87 & 130.93 & 0.17 & 0.18 & 0.14 & 0.16 \\
\hline $\mathrm{S}_{2}-\mathrm{P}_{0} \mathrm{Mg}_{1}$ & 836.00 & 748.02 & 903.00 & 825.00 & 139.20 & 139.10 & 0.19 & 0.19 & 0.15 & 0.17 \\
\hline $\mathrm{S}_{2}-\mathrm{P}_{0} \mathrm{Mg}_{2}$ & 836.00 & 748.02 & 903.00 & 825.00 & 160.93 & 163.93 & 0.22 & 0.22 & 0.18 & 0.20 \\
\hline $\mathrm{S}_{2}-\mathrm{P}_{1} \mathrm{Mg}_{0}$ & 836.00 & 748.02 & 903.00 & 825.00 & 131.17 & 174.00 & 0.18 & 0.23 & 0.15 & 0.21 \\
\hline $\mathrm{S}_{2}-\mathrm{P}_{1} \mathrm{Mg}_{1}$ & 836.00 & 748.02 & 903.00 & 825.00 & 171.93 & 196.90 & 0.23 & 0.26 & 0.19 & 0.24 \\
\hline $\mathrm{S}_{2}-\mathrm{P}_{1} \mathrm{Mg}_{2}$ & 836.00 & 748.02 & 903.00 & 825.00 & 203.50 & 216.90 & 0.27 & 0.29 & 0.23 & 0.26 \\
\hline $\mathrm{S}_{2}-\mathrm{P}_{2} \mathrm{Mg}_{0}$ & 836.00 & 748.02 & 903.00 & 825.00 & 369.40 & 377.57 & 0.49 & 0.50 & 0.41 & 0.46 \\
\hline $\mathrm{S}_{2}-\mathrm{P}_{2} \mathrm{Mg}_{1}$ & 836.00 & 748.02 & 903.00 & 825.00 & 424.47 & 397.97 & 0.57 & 0.53 & 0.47 & 0.48 \\
\hline $\mathrm{S}_{2}-\mathrm{P}_{2} \mathrm{Mg}_{2}$ & 836.00 & 748.02 & 903.00 & 825.00 & 468.07 & 445.10 & 0.63 & 0.60 & 0.52 & 0.54 \\
\hline $\mathrm{S}_{2}-\mathrm{P}_{3} \mathrm{Mg}_{0}$ & 836.00 & 748.02 & 903.00 & 825.00 & 377.33 & 370.27 & 0.50 & 0.50 & 0.42 & 0.45 \\
\hline $\mathrm{S}_{2}-\mathrm{P}_{3} \mathrm{Mg}_{1}$ & 836.00 & 748.02 & 903.00 & 825.00 & 378.70 & 387.83 & 0.51 & 0.52 & 0.42 & 0.47 \\
\hline $\mathrm{S}_{2}-\mathrm{P}_{3} \mathrm{Mg}_{2}$ & 836.00 & 748.02 & 903.00 & 825.00 & 440.63 & 411.13 & 0.59 & 0.55 & 0.49 & 0.50 \\
\hline $\mathrm{S}_{3}-\mathrm{P}_{0} \mathrm{Mg}_{0}$ & 1067.00 & 981.00 & 1164.00 & 1070.00 & 326.60 & 335.33 & 0.33 & 0.34 & 0.28 & 0.31 \\
\hline $\mathrm{S}_{3}-\mathrm{P}_{0} \mathrm{Mg}_{1}$ & 1067.00 & 981.00 & 1164.00 & 1070.00 & 382.80 & 351.17 & 0.39 & 0.36 & 0.33 & 0.33 \\
\hline $\mathrm{S}_{3}-\mathrm{P}_{0} \mathrm{Mg}_{2}$ & 1067.00 & 981.00 & 1164.00 & 1070.00 & 447.17 & 451.73 & 0.46 & 0.46 & 0.38 & 0.42 \\
\hline $\mathrm{S}_{3}-\mathrm{P}_{1} \mathrm{Mg}_{0}$ & 1067.00 & 981.00 & 1164.00 & 1070.00 & 432.30 & 406.97 & 0.44 & 0.41 & 0.37 & 0.38 \\
\hline $\mathrm{S}_{3}-\mathrm{P}_{1} \mathrm{Mg}_{1}$ & 1067.00 & 981.00 & 1164.00 & 1070.00 & 492.53 & 496.43 & 0.50 & 0.51 & 0.42 & 0.46 \\
\hline $\mathrm{S}_{3}-\mathrm{P}_{1} \mathrm{Mg}_{2}$ & 1067.00 & 981.00 & 1164.00 & 1070.00 & 564.50 & 517.90 & 0.58 & 0.53 & 0.48 & 0.48 \\
\hline $\mathrm{S}_{3}-\mathrm{P}_{2} \mathrm{Mg}_{0}$ & 1067.00 & 981.00 & 1164.00 & 1070.00 & 386.40 & 418.17 & 0.39 & 0.43 & 0.33 & 0.39 \\
\hline $\mathrm{S}_{3}-\mathrm{P}_{2} \mathrm{Mg}_{1}$ & 1067.00 & 981.00 & 1164.00 & 1070.00 & 405.77 & 509.83 & 0.41 & 0.52 & 0.35 & 0.48 \\
\hline $\mathrm{S}_{3}-\mathrm{P}_{2} \mathrm{Mg}_{2}$ & 1067.00 & 981.00 & 1164.00 & 1070.00 & 444.43 & 533.57 & 0.45 & 0.54 & 0.38 & 0.50 \\
\hline $\mathrm{S}_{3}-\mathrm{P}_{3} \mathrm{Mg}_{0}$ & 1067.00 & 981.00 & 1164.00 & 1070.00 & 338.50 & 340.47 & 0.35 & 0.35 & 0.29 & 0.32 \\
\hline $\mathrm{S}_{3}-\mathrm{P}_{3} \mathrm{Mg}_{1}$ & 1067.00 & 981.00 & 1164.00 & 1070.00 & 396.07 & 379.83 & 0.40 & 0.39 & 0.34 & 0.35 \\
\hline $\mathrm{S}_{3}-\mathrm{P}_{3} \mathrm{Mg}_{2}$ & 1067.00 & 981.00 & 1164.00 & 1070.00 & 411.77 & 413.20 & 0.42 & 0.42 & 0.35 & 0.39 \\
\hline
\end{tabular}

It was seen that when water amounts increased, yields generally increased. However, related to fertilizer treatments, the difference among yields didn't occur similar to the other irrigation parts. The effect of water on fertilizer and fertilizer consumption by applied water are similar to each other. The differences between study years were not statistically significant. The highest yield was obtained from an irrigation plot on which $\mathrm{P}_{1} \mathrm{Mg}_{2}$ fertilizer combination applied to reach these yield values, total applied water amounts were 1067 and $981 \mathrm{~mm}$ in the first and second year. These results produced the highest IWTE and WCE results. The reason for varying yields from fertilizer combinations under the same water regime was that magnesium increases phosphorus uptake when water is increased. In the same irrigation parts, 326.6 and $335.33 \mathrm{~kg} \mathrm{da}^{-1}$ yield were obtained from $\mathrm{P}_{0} \mathrm{Mg}_{0}$ fertilizer combination. IWTE and WCE values with respect to years were $0.33,0.34$ and $0.28,0.31 \mathrm{~kg} \mathrm{~m}^{-3}$ (Tab. 2)

When irrigation parts or fertilizer combinations were observed, the interactions between irrigation water treat- 
Tab. 3. Rational water consumption inadequacy compared with rational yield decrease in 2006 and 2007

\begin{tabular}{|c|c|c|c|c|c|c|c|c|c|c|c|c|c|c|c|c|}
\hline \multirow[t]{2}{*}{$\begin{array}{l}\text { Irrigation } \\
\text { parts }\end{array}$} & \multicolumn{2}{|c|}{$Y_{2}$} & \multicolumn{2}{|c|}{$\mathrm{ET}_{\mathrm{a}}$} & \multicolumn{2}{|c|}{$Y_{a} / Y_{m}$} & \multicolumn{2}{|c|}{$1-\left(Y_{a} / Y_{m}\right)$} & \multicolumn{2}{|c|}{$\mathrm{ET}_{\mathrm{a}} / \mathrm{ET}_{\mathrm{m}}$} & \multicolumn{2}{|c|}{$\begin{array}{c}1-\left(\mathrm{ET}_{\mathrm{a}} /\right. \\
\left.\mathrm{ET}_{\mathrm{m}}\right)^{-}\end{array}$} & \multicolumn{2}{|c|}{$\begin{array}{c}\text { Amounts of } \\
\text { irrigation water } \\
(\mathrm{mm})\end{array}$} & \multicolumn{2}{|c|}{$\begin{array}{c}\text { Water } \\
\text { Economics } \\
(\%)\end{array}$} \\
\hline & 2006 & 2007 & 2006 & 2007 & 2006 & 2007 & 2006 & 2007 & 2006 & 2007 & 2006 & 2007 & 2006 & 2007 & 2006 & 2007 \\
\hline $\mathrm{S}_{1}-\mathrm{P}_{0} \mathrm{Mg}_{0}$ & 111.1 & 107.4 & 648.00 & 567.00 & 0.32 & 0.32 & 0.68 & 0.68 & 0.56 & 0.53 & 0.44 & 0.47 & 598.00 & 507.98 & 0.44 & 0.48 \\
\hline $\mathrm{S}_{1}-\mathrm{P}_{0} \mathrm{Mg}_{1}$ & 126.8 & 125.5 & 648.00 & 567.00 & 0.37 & 0.38 & 0.63 & 0.62 & 0.56 & 0.53 & 0.44 & 0.47 & 598.00 & 507.98 & 0.44 & 0.48 \\
\hline $\mathrm{S}_{1}-\mathrm{P}_{0} \mathrm{Mg}_{2}$ & 132.1 & 131.9 & 648.00 & 567.00 & 0.38 & 0.39 & 0.63 & 0.61 & 0.56 & 0.53 & 0.44 & 0.47 & 598.00 & 507.98 & 0.44 & 0.48 \\
\hline $\mathrm{S}_{1}-\mathrm{P}_{1} \mathrm{Mg}_{0}$ & 134.7 & 121.3 & 648.00 & 567.00 & 0.39 & 0.36 & 0.61 & 0.64 & 0.56 & 0.53 & 0.44 & 0.47 & 598.00 & 507.98 & 0.44 & 0.48 \\
\hline $\mathrm{S}_{1}-\mathrm{P}_{1} \mathrm{Mg}_{1}$ & 140.8 & 140.2 & 648.00 & 567.00 & 0.41 & 0.42 & 0.59 & 0.58 & 0.56 & 0.53 & 0.44 & 0.47 & 598.00 & 507.98 & 0.44 & 0.48 \\
\hline $\mathrm{S}_{1}-\mathrm{P}_{1} \mathrm{Mg}_{2}$ & 162.0 & 156.5 & 648.00 & 567.00 & 0.47 & 0.47 & 0.53 & 0.53 & 0.56 & 0.53 & 0.44 & 0.47 & 598.00 & 507.98 & 0.44 & 0.48 \\
\hline $\mathrm{S}_{1}-\mathrm{P}_{2} \mathrm{Mg}_{0}$ & 207.8 & 219.8 & 648.00 & 567.00 & 0.60 & 0.66 & 0.40 & 0.34 & 0.56 & 0.53 & 0.44 & 0.47 & 598.00 & 507.98 & 0.44 & 0.48 \\
\hline $\mathrm{S}_{1}-\mathrm{P}_{2} \mathrm{Mg}_{1}$ & 217.4 & 239.6 & 648.00 & 567.00 & 0.63 & 0.7 & 0.37 & 0.28 & 0.56 & 0.53 & 0.44 & 0.47 & 598.00 & 507.98 & 0.44 & 0.48 \\
\hline $\mathrm{S}_{1}-\mathrm{P}_{2} \mathrm{Mg}_{2}$ & 235.1 & 250.2 & 648.00 & 567.00 & 0.68 & 0.7 & 0.32 & 0.25 & 0.56 & 0.53 & 0.44 & 0.47 & 598.00 & 507.98 & 0.44 & 0.48 \\
\hline $\mathrm{S}_{1}-\mathrm{P}_{3} \mathrm{Mg}_{0}$ & 188.0 & 219.9 & 648.00 & 567.00 & 0.54 & 0.66 & 0.46 & 0.34 & 0.56 & 0.53 & 0.44 & 0.47 & 598.00 & 507.98 & 0.44 & 0.48 \\
\hline $\mathrm{S}_{1}-\mathrm{P}_{3} \mathrm{Mg}_{1}$ & 243.5 & 243.4 & 648.00 & 567.00 & 0.71 & 0.73 & 0.29 & 0.27 & 0.56 & 0.53 & 0.44 & 0.47 & 598.00 & 507.98 & 0.44 & 0.48 \\
\hline $\mathrm{S}_{1}-\mathrm{P}_{3} \mathrm{Mg}_{2}$ & 345.2 & 334.2 & 648.00 & 567.00 & 1.00 & 1.00 & 0.00 & 0.00 & 0.56 & 0.53 & 0.44 & 0.47 & 598.00 & 507.98 & 0.44 & 0.48 \\
\hline $\mathrm{S}_{2}-\mathrm{P}_{0} \mathrm{Mg}_{0}$ & 128.87 & 130.93 & 903.00 & 825.00 & 0.28 & 0.29 & 0.72 & 0.71 & 0.78 & 0.77 & 0.22 & 0.23 & 836.00 & 748.00 & 0.22 & 0.24 \\
\hline $\mathrm{S}_{2}-\mathrm{P}_{0} \mathrm{Mg}_{1}$ & 139.20 & 139.10 & 903.00 & 825.00 & 0.30 & 0.31 & 0.70 & 0.69 & 0.78 & 0.77 & 0.22 & 0.23 & 836.00 & 748.00 & 0.22 & 0.24 \\
\hline $\mathrm{S}_{2}-\mathrm{P}_{0} \mathrm{Mg}_{2}$ & 160.93 & 163.93 & 903.00 & 825.00 & 0.34 & 0. & 0.66 & 0.63 & 0.78 & 0.77 & 0.22 & 0.23 & 836.00 & 748.00 & 0.22 & 0.24 \\
\hline $\mathrm{S}_{2}-\mathrm{P}_{1} \mathrm{Mg}_{0}$ & 131.17 & & & & & & & & & & & & 836.00 & & 0.22 & 0.24 \\
\hline $\mathrm{S}_{2}-\mathrm{P}_{1} \mathrm{Mg}_{1}$ & 171.93 & 196.90 & 903.00 & 825.00 & 0.37 & 0.44 & 0.63 & 0.56 & 0.78 & 0.77 & 0.22 & 0.23 & 836.00 & 748.00 & 0.22 & 0.24 \\
\hline $\mathrm{S}_{2}-\mathrm{P}_{1} \mathrm{Mg}_{2}$ & 203.50 & 216.90 & 903.00 & 825.00 & 0.43 & 0.49 & 0.57 & 0.51 & 0.78 & 0.77 & 0.22 & 0.23 & 836.00 & 748.00 & 0.22 & 0.24 \\
\hline $\mathrm{S}_{2}-\mathrm{P}_{2} \mathrm{Mg}_{0}$ & 369.40 & 377.57 & 903.00 & 825.00 & 0.79 & 0.85 & 0.21 & 0.15 & 0.78 & 0.77 & 0.22 & 0.23 & 836.00 & 748.00 & 0.22 & 0.24 \\
\hline $\mathrm{S}_{2}-\mathrm{P}_{2} \mathrm{Mg}_{1}$ & 424.47 & 397.97 & 903.00 & 825.00 & 0.91 & 0.89 & 0.09 & 0.11 & 0.78 & 0.77 & 0.22 & 0.23 & 836.00 & 748.00 & 0.22 & 0.24 \\
\hline $\mathrm{S}_{2}-\mathrm{P}_{2} \mathrm{Mg}_{2}$ & 468.07 & 445.10 & 903.00 & 825.00 & 1.00 & 1.00 & 0.00 & 0.00 & 0.78 & 0.77 & 0.22 & & 836.00 & & 0.22 & 0.24 \\
\hline $\mathrm{S}_{2}-\mathrm{P}_{3} \mathrm{Mg}_{0}$ & 377.33 & 370.27 & 903.00 & 825.00 & 0.81 & 0.83 & 0.19 & 0.17 & 0.78 & 0.77 & 0.22 & 0.23 & 836.00 & 748.00 & 0.22 & 0.24 \\
\hline $\mathrm{S}_{2}-\mathrm{P}_{3} \mathrm{Mg}_{1}$ & 378.70 & 387.83 & 903.00 & 825.00 & 0.81 & 0.87 & 0.19 & 0.13 & 0.78 & 0.77 & 0.22 & 0.23 & 836.00 & 748.00 & 0.22 & 0.24 \\
\hline $\mathrm{S}_{2}-\mathrm{P}_{3} \mathrm{Mg}_{2}$ & 440.63 & 411.13 & 903.00 & 825.00 & 0.94 & 0.92 & 0.06 & 0.08 & 0.78 & 0.77 & 0.22 & 0.23 & 836.00 & 748.00 & 0.22 & 0.24 \\
\hline $\mathrm{S}_{3}-\mathrm{P}_{0} \mathrm{Mg}_{0}$ & 326.60 & 335.33 & 1164.00 & 1070.00 & 0.58 & 0.63 & 0.42 & 0.37 & 1.00 & 1.00 & 0.00 & 0.00 & 1067.00 & 981.00 & 0.00 & 0.00 \\
\hline $\mathrm{S}_{3}-\mathrm{P}_{0} \mathrm{Mg}_{1}$ & 382.80 & 351.17 & 1164.00 & 1070.00 & 0.68 & 0.66 & 0.32 & 0.34 & 1.00 & 1.00 & 0.00 & 0.00 & 1067.00 & 981.00 & 0.00 & 0.00 \\
\hline $\mathrm{S}_{3}-\mathrm{P}_{0} \mathrm{Mg}_{2}$ & 447.17 & 451.73 & 1164.00 & 1070.00 & 0.79 & 0.85 & 0.21 & 0.15 & 1.00 & 1.00 & 00 & 0.00 & 1067.00 & 981.00 & 0.00 & 0.00 \\
\hline $\mathrm{S}_{3}-\mathrm{P}_{1} \mathrm{Mg}_{0}$ & 432.30 & 406.97 & 1164.00 & 1070.00 & 0.77 & 0.76 & 0.23 & 0.24 & 1.00 & 1.00 & 0.00 & 0.00 & 1067.00 & 981.00 & 0.00 & 0.00 \\
\hline $\mathrm{S}_{3}-\mathrm{P}_{1} \mathrm{Mg}_{1}$ & 492.53 & 496.43 & 1164.00 & 1070.00 & 0.87 & 0.93 & 0.13 & 0.07 & 1.00 & 1.00 & 0.00 & 0.00 & 1067.00 & 981.00 & 0.00 & 0.00 \\
\hline $\mathrm{S}_{3}-\mathrm{P}_{1} \mathrm{Mg}_{2}$ & 564.50 & 517.90 & 1164.00 & 1070.00 & 1.00 & 0.97 & 0.00 & 0.03 & 1.00 & 1.00 & 0.00 & 0.00 & 1067.00 & 981.00 & 0.00 & 0.00 \\
\hline $\mathrm{S}_{3}-\mathrm{P}_{2} \mathrm{Mg}_{0}$ & 386.40 & 418.17 & 1164.00 & 1070.00 & 0.68 & 0.78 & 0.32 & 0.22 & 1.00 & 1.00 & 0.00 & 0.00 & 1067.00 & 981.00 & 0.00 & 0.00 \\
\hline $\mathrm{S}_{3}-\mathrm{P}_{2} \mathrm{Mg}_{1}$ & 405.77 & 509.83 & 1164.00 & 1070.00 & 0.72 & 0.96 & 0.28 & 0.04 & 1.00 & 1.00 & 0.00 & 0.00 & 1067.00 & 981.00 & 0.00 & 0.00 \\
\hline $\mathrm{S}_{3}-\mathrm{P}_{2} \mathrm{Mg}_{2}$ & 444.43 & 533.57 & 1164.00 & 1070.00 & 0.79 & 1.00 & 0.21 & 0.00 & 1.00 & 1.00 & 0.00 & 0.00 & 1067.00 & 981.00 & 0.00 & 0.00 \\
\hline $\mathrm{S}_{3}-\mathrm{P}_{3} \mathrm{Mg}_{0}$ & 338.50 & 340.47 & 1164.00 & 1070.00 & 0.60 & 0.64 & 0.40 & 0.36 & 1.00 & 1.00 & 0.00 & 0.00 & 1067.00 & 981.00 & 0.00 & 0.00 \\
\hline $\mathrm{S}_{3}-\mathrm{P}_{3} \mathrm{Mg}_{1}$ & 396.07 & 379.83 & 1164.00 & 1070.00 & 0.70 & 0.71 & 0.30 & 0.29 & 1.00 & 1.00 & 0.00 & 0.00 & 1067.00 & 981.00 & 0.00 & 0.00 \\
\hline $\mathrm{S}_{3}-\mathrm{P}_{3} \mathrm{Mg}_{2}$ & 411.77 & 413.20 & 1164.00 & 1070.00 & 0.73 & 0.77 & 0.27 & 0.23 & 1.00 & 1.00 & 0.00 & 0.00 & 1067.00 & 981.00 & 0.00 & 0.00 \\
\hline
\end{tabular}

ment efficiency (IWTE) and WCE values were due to both water and fertilizer. The highest yield factor among fertilizer combinations was based on $\mathrm{Mg}_{2}$ doses to which 8 $\mathrm{kg} \mathrm{Mg}$ sulfate fertilizer was applied. In arid and semi-arid regions where the water resources are limited, $\mathrm{P}_{3} \mathrm{Mg}_{2}$ fertilizer combination is suggested for soybean plant for $\mathrm{I}_{1}$ water level. In similar regions, at $\mathrm{I}_{2}$ water level with $33 \%$ water limitation the highest yield was obtained from $\mathrm{P}_{2} \mathrm{Mg}$ fertilizer combination. However, in arid and semi-arid regions, on parts without any limitations on resources, the highest yield was obtained from $\mathrm{P}_{1} \mathrm{Mg}_{2}$ fertilizer combination (Tab. 2).

The findings of the present study are consistent with those of other studies in the literature. Simsek et al. (2005), investigated the water and yield relationship of soybean irrigated in different irrigation sequences with sprinkling- dripping irrigation systems; they reported that a $10 \%$ reduction in water consumption caused a $5.2 \%$ reduction in yield. In a study of water-yield between relationships in combined crop-soybean cultivation systems. 


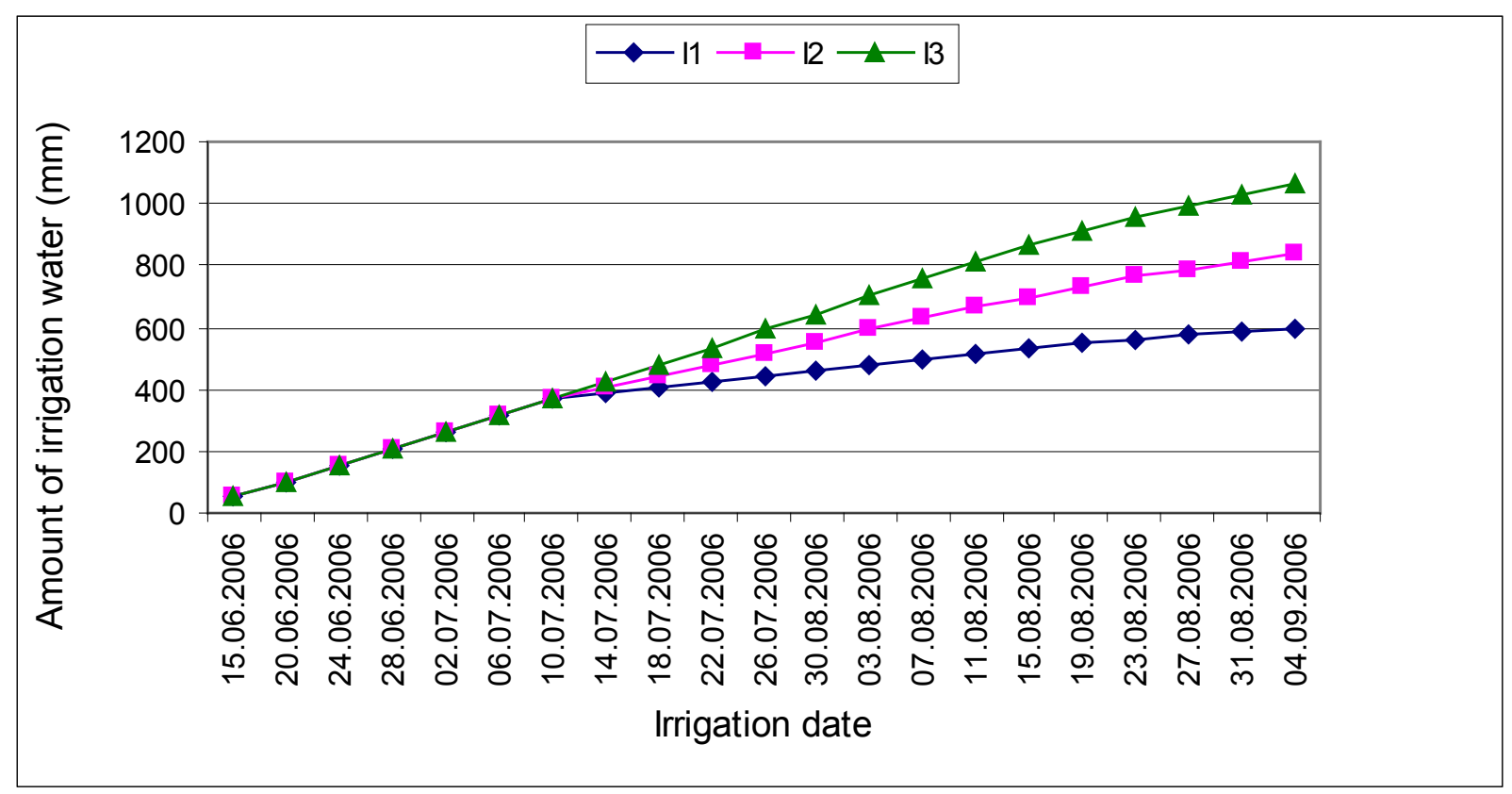

Fig. 1. Amounts of irrigation water and dates in 2006

Simsek et al. (2001), reported that the highest irrigation water treatment efficiency (IWTE) and water usage efficiency (WUE) values were $0.87-0.77 \mathrm{~kg} \mathrm{~m}^{-3}$, respectively in a 2 Crop / 1 soybean cultivation system and the lowest IWTE and WUE values were $0.51-0.50 \mathrm{~kg} \mathrm{~m}^{-3}$ in a 1 Crop/2 Soybean cultivation system.

Yazar et al. (1989), reported a significant linear relationship between grain yield and seasonal water consumption in a study of water-yield relationship in soy as sec- ond yield in Cukurova conditions. Vearela (1998), found that when water stress created at different times of soy increased from $20 \%$ to $40 \%$, dry matter amount decreased between 25-34\%. Larry and Spurlock (1993), determined irrigation water usage efficiency (IWUE) values between $1.3-5.6 \mathrm{~kg} \mathrm{ha}^{-1} \mathrm{~mm}^{-1}$ in a study of soybean in the USA.

Water-yield relations and yield values at different irrigation levels varied between the two study years. However, plant water consumptions showed different $\mathrm{I}_{1}, \mathrm{I}_{2}$ and $\mathrm{I}_{3}$

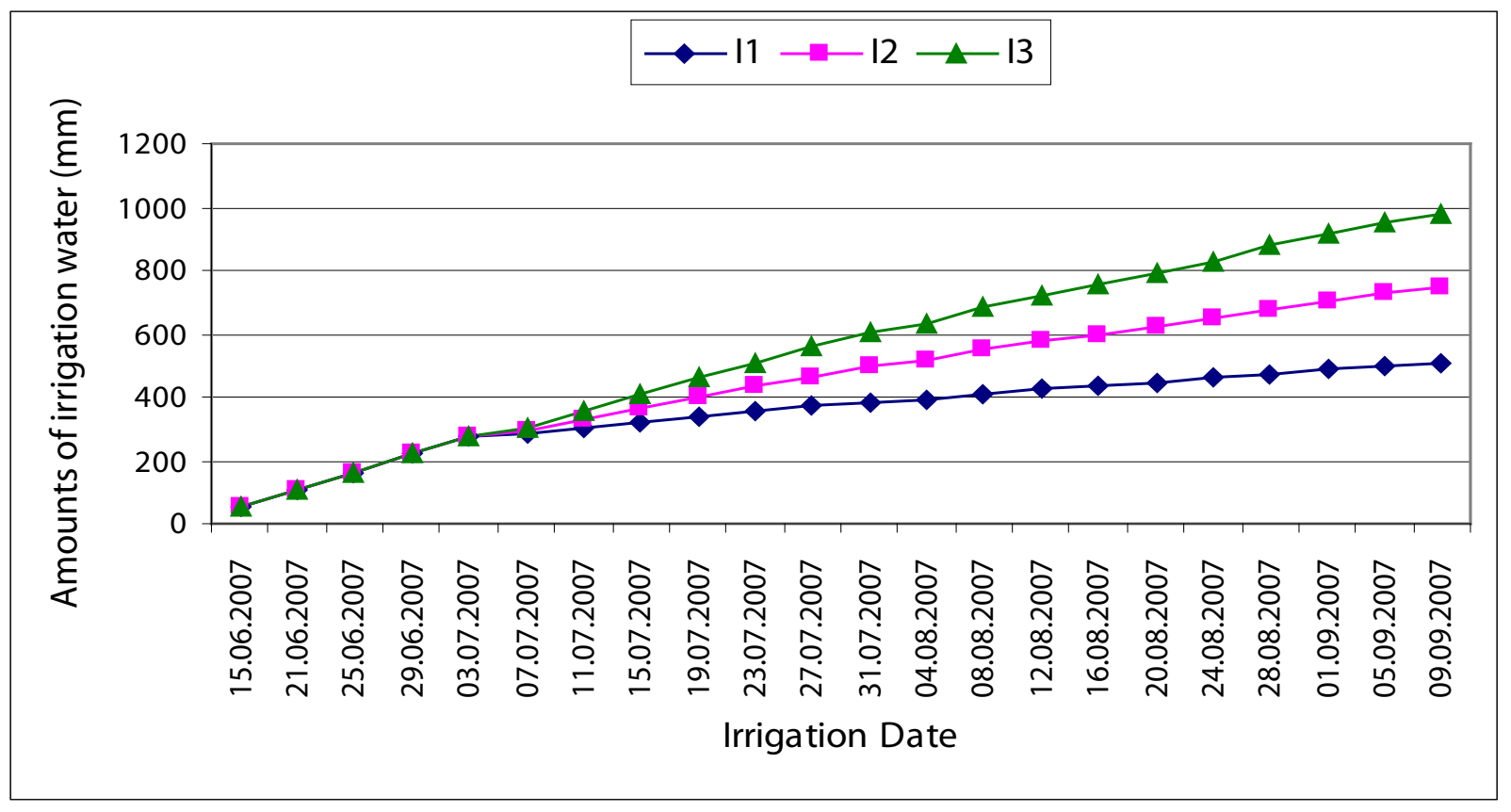

Fig. 2. Amounts of irrigation water and dates in 2007 
irrigations. Generally, these differences were reflected in yields. The differing yields were related to fertilizer doses ( $\mathrm{P}$ and $\mathrm{Mg}$ ). Rational yield values on $\mathrm{I}_{1}$ irrigation plots increased with $\mathrm{P}$ and $\mathrm{Mg}$ increase. Reaction of the same fertilizer combinations showed differences for $\mathrm{I}_{2}$ and $\mathrm{I}_{3}$ and this rate obtained from $\mathrm{P}_{2} \mathrm{Mg}_{2}$ for $\mathrm{I}_{2}$ irrigation part and from $\mathrm{P}_{1} \mathrm{Mg}_{2}$ for $\mathrm{I}_{3}$ part. Bayrak (1989), reported on a 3-year study of $\mathrm{P}$-water relation and water consumption of soybean in Bafra Plain. Four different water levels and four different $\mathrm{P}\left(0-4-8-12 \mathrm{~kg} \mathrm{da}^{-1} \mathrm{P}_{2} \mathrm{O}_{5}\right)$ levels were applied. The highest average yield $\left(349 \mathrm{~kg} \mathrm{da}^{-1}\right)$ was obtained from I irrigation plots with $\mathrm{P}_{2}$ fertilizer part and $414.6 \mathrm{~mm}$ water. In a study examining the effect of water, fertilizer and bacteria inoculation on soybean yield, Vasiliu and Pascaru (1977), found that NPP (Nitrogen-Phosphorus-Potassium) fertilizers with water increased soybean yield. Yavada (1980), applied $\mathrm{P}_{2} \mathrm{O}_{5}$ at $0-80 \mathrm{~kg} \mathrm{da}^{-1}$ and $\mathrm{N}$ at $0-100 \mathrm{~kg}$ $\mathrm{da}^{-1}$ at three soil moisture levels, and found that irrigation and fertilization increased seed yield. In a study, water and chemical fertilizers were effect of on the yield of 'Clark 63' soybean type. Saadi and Yazdi-Samadi (1978), reported that water and phosphorus fertilizer in combination increased soybean grains, grain number of soybean, number of pods and yield.

When water savings examined, $44 \%$ saving provided on $\mathrm{I}_{1}$ part and all fertilizer combination and $56 \%$ of water applied. However, rational yields and rational yield decreases varied according to fertilizer combinations. Rational yield increased with increased $\mathrm{P}$ and $\mathrm{Mg}$ doses, whereas rational yield decreases varied according to fertilizer combinations. Rational yield was 1 on $\mathrm{P}_{3} \mathrm{Mg}_{2}$ fertilizer combination, from which the highest yield was obtained and rational yield reduction was not observed (Tab. 3). Water saving was $22 \%$ for all fertilizer combinations on $\mathrm{I}_{2}$ irrigation plots and $78 \%$ of water applied to it. However, rational yield and rational yield decreases varied according to fertilizer combinations. Rational yield was 1 on $\mathrm{P}_{2} \mathrm{Mg}_{2}$ fertilizer combination, from which the highest yield was obtained, and rational yield decreases were not observed.

Rational yield and rational yield decrease values varied according to fertilizer combinations on complete irrigation parts $\left(\mathrm{I}_{3}\right)$, on which $100 \%$ irrigation quota was applied. Rational yield was 1 with $\mathrm{P}_{1} \mathrm{Mg}_{2}$ fertilizer combination, from which the highest yield was obtained, and rational yield reduction was not observed. It is thought that this was related to maximum water usage and consumption (Tab. 3). Whitt and Van Bavel (1955), note that $300 \mathrm{~kg}$ water is required to obtain $1 \mathrm{~kg}$ dry matter from soybean. Fernandez et al. (1978), applied four water levels to Amsoy soybean type and found that the yield obtained from the minimum irrigated part was $1.4 \mathrm{t} \mathrm{ha}^{-1}$ and $2.5 \mathrm{t}$ $\mathrm{ha}^{-1}$ from the maximum irrigated part. Sarma et al. (1976), examined the effect of irrigation on capsule and grain-filling time during flowering time on two different soy types. Irrigation during flowering produced a yield of $2.46 \mathrm{com}-$ pared with 2.37 as a result of irrigation during grain-filling time and $1.9 \mathrm{t} \mathrm{ha}^{-1}$ obtained from dry parts.

Saenko (1977), found that water treatments of $60 \%$, $70 \%$ and $80 \%$ of field capacity produced average yields of $1.42,1.93$ and $2.53 \mathrm{t} \mathrm{ha}^{-1}$ respectively, compared with $0.71 \mathrm{t} \mathrm{ha}^{-1}$ yield obtained non-irrigated parts. In another study of researches, they found that the highest yield was $4.25 \mathrm{tha}^{-1}$ under irrigated conditions and $1.69 \mathrm{tha}^{-1}$ under dry conditions. Simiciklas et al. (1989), reported that, in their pot research examining the effect of drought stress at different generative periods on plant growth and yield, water inadequacy inhibited plant nutrient transformation and, so seed yield and weight of 1000 grain are decreased. Muandemele and Doto (1988), conducted a field experiment on 30 soybean types, drought was decreased soybean plant, seed bean rate grain yield and exposure to drought resulted in smaller grain-size and reduced germination power. Simsek et al. (2001), found that a 10\% reduction in water consumption resulted in a 5.2\% lower yield; the lowest irrigation water treatment efficiency (IWTE) and water usage efficiency (WUE) values were 1.98-2.10 respectively and the highest values were $5.3-6.44 \mathrm{~kg} \mathrm{ha}^{-1}$ $\mathrm{mm}^{-1}$. The findings of the present study are consistent with those of other researchers.

\section{Conclusions}

According to the research findings, increased irrigation water, $\mathrm{P}$ and $\mathrm{Mg}$ treatments produced higher yields. The highest yield was obtained from $\mathrm{I}_{3}(100)$ irrigation part and $\mathrm{P}_{1} \mathrm{Mg}_{2}\left(564.50 \mathrm{~kg} \mathrm{da}^{-1}\right)$ treatment in the first year and from $\mathrm{P}_{2} \mathrm{Mg}_{2}\left(533.57 \mathrm{~kg} \mathrm{da}^{-1}\right)$ treatment on the same irrigation part in the second year (2007). The lowest yield was obtained from irrigation parts subjected to water stress. Yield increased by applying $\mathrm{Mg}$ to the soil, resulting in much higher yields compared with global soybean yields. $\mathrm{Mg}$ can play an important role leveling increasing yields in cases where water and fertilizer treatments must be reduced.

It is understood that, with the start of irrigation on Harran Plain, soybean can be included in sowing turn. The findings of this study demonstrate that high quality yields can be obtained while reducing water and fertilizer inputs; these results should be conveyed to producers and the findings may inform future studies related to soybean in this region.

\section{References}

Bayrak F (1989). Phosphorus-water relationships and water consumption soybean in Bafra plain. Generel Directorate of Rural Service. General Research Institute of Samsun Bullt. General Publication No: 50, Report Series No: 44, Samsun.

Doorenbos J, Kassam AH (1988). Yield Response to Water. FAO Irrig. Drain Paper 33, Rome. 
190

FAO (1979). Faostat, Rome, Italy.

Fernandez JL (1978). Growth and Yield of Soybean Crop at Different Levels of Irrigation. Departo Mento. Nacional de Plantes Oleaginosaso INIA Cordoba Spain.

Gercek S, Simsek M, Kirnak H, Boydak E (2003). The Effect of Water Consumption of soybean of different irragation methods Harran Plain Condition in Sanliurfa. Harran Univ Journal of Agriculture Faculty 7(1-2):61-68.

Howell TA, Cuenca HA, Solomon KH (1990). Crop Yield Response. Management of Farm Irrigation Systems. Trans. ASAE Monograph Chap S. USA.

Kadayifci A (1996). Sunflower Water-Yield Relationships. AU. Institute of Natural Science, PhD Thesis, p. 12-89.

Larry GH, Spurlock R (1993). Timing of Furrow Irrigation Termination for Determinate Soybean on Clay Soil. Argon. J 85(6):1103-1108.

Muandemele OD, Doto A (1988). Evaluation of Soybean Lines for Drought Tolerance and the Influence of Water Availability on Cookability. Turialba 38:194-197.

Ozkara MM (1991). Second-crop soya Menemen Region Water Consumption. Menemen Agricultural Research Institute Publications. Publ No: 170/R-11, Turkey.

Tulucu K (1985). Deficit Irrigation Practice for Agricultural Irrigation, Water-Production Function Concept and Use of Resources The Best Way. Journal of Natural Science 9(1):1224.

TSMS-Turkish State Meteorological Service (2006). Bulletin of climate parameters the Sanliurfa. Ankara.

TSMS-Turkish State Meteorological Service (2007). Bulletin of climate parameters the Sanliurfa. Ankara.

Sanliurfa Rural Services Research Institute (2001). Water Year Hydrometeorological Observation Data. Bullt. No:79, Sanliurfa.

Saadi K, Yazdi-Samadi B (1978). Effect of Irrigation and Chemical Fertilizers of Soybean. Iranian Journal of Agricultural Science 21(4):214-219.

Saenko NP (1977). Effect of Irrigation on Different Soybean Cultivars in Sarpin Depression of Kalmyk, USSR.

Sarma SR, Raghu JS, Choubey SD (1976). Response of Soybean Varieties Irrigation, Given at Different Growth Stage Under Normal and Late Sown Conditions. Indian Journal of Agronomy 21(4):412-415.
Shariatmadari H, Mermut AR (1999). Magnesium and Silicon Induced Phosphate Desorption in Smectic, Palygorskite and Sepiolite-Calcite Systems. Soil Science Society of America Journal 63:1167-1173.

Simiciklas RG, Carrillo SP, Agudelo DO (1989). Evaluation of Soybean Cultivars With Different Growth Habits Accepting to Irrigation Level. Acta-Agronomical 38:7-22.

Simsek M, Boydak E, Gercek S, Kirnak H (2001). Determination of the relationship between soybean yield in irrigated water Drip Irrigation Techniques -Sequence interval sprinkler Harran Plain Condition. AU. Journal of Agric Faculty 7(3):88-93.

Simsek M, Silbir Y, Gercek S, Boydak E, Kasap Y (2005). Corn-Soybean Yield and Land Equivalent Ratio Together Oct. System Water-relations Determination. Ankara Univ. Journal Agric Faculty 2:147-153.

Vasiliu M, Pascaru E (1977). The Influence of Irrigation and Fertilizers upon Soybean Yield and Quality in the Braila Plain. Research Institute for Cereals and Technical Plants Fundulea 37:335-42.

Vearela BD (1998). Deficit Irrigation During the Reproductive Stages of Soybean (Glycine max L.). Merrill Philippines Uni. Vol. 97. Collage Laguna, Los Banos.

Whitt DM CHM van Bavel CHM (1955). Irrigation of Tobacco Peanuts and Soybean, pp. 376-380. In: Water. The Year Book of Agriculture U.S.D.A.

Yazar A, Cevik B, Tekinel O, Tülücü K, Kamber R, Basbug R (1989). Sprinkler Method Second Crop Soybean Yield Evapotaranspirasyon Cukurova Conditions Determining the Relationships. TUBITAK-TOAG (551).

Yaron D (1971). Estimation and Use of Water Production funtions in Crops. Journal of the Irrigation Drain Div Proc. Trans 5:291-302.

Yavada BS (1980). Studies on the Effect of Inoculation N and Fertilization and Moisture Regimes on the Soybean Production, p. 353-391. In: Hardy RWF, Gibson AH (Eds.). A treatise on dinitrogen fixation. Sect. IV. Agronomy and Ecology. John Wiley and Sons, NY. 